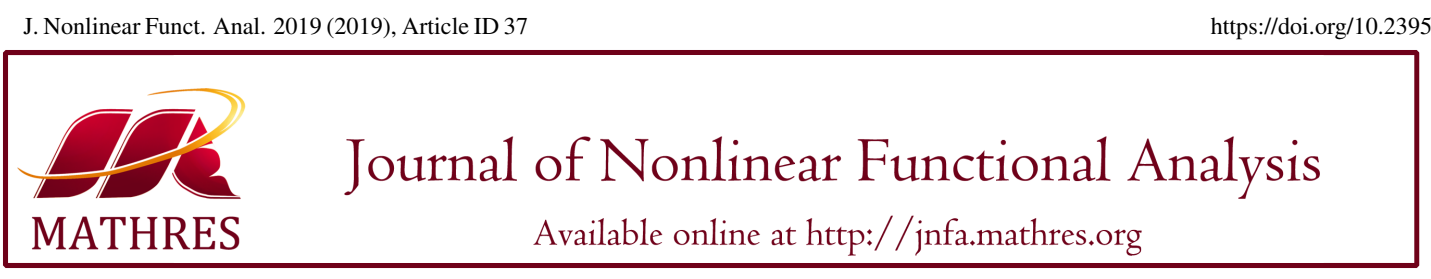

\title{
SINGULARITIES OF ATTRACTIVE AND REPULSIVE TYPE TO FOURTH-ORDER NEUTRAL LIÉNARD EQUATIONS WITH TIME-DEPENDENT DEVIATING ARGUMENTS
}

\author{
YUN XIN $^{1}$, ZHIBO CHENG $^{2,3, *}$ \\ ${ }^{1}$ College of Computer Science and Technology, Henan Polytechnic University, Jiaozuo 454000, China \\ ${ }^{2}$ School of Mathematics and Information Science, Henan Polytechnic University, Jiaozuo 454000, China \\ ${ }^{3}$ Department of Mathematics, Sichuan University, Chengdu 610064, China
}

\begin{abstract}
In this paper, we are concerned with the existence of a positive periodic solution for a kind of fourth-order neutral singular Liénard equation with time-dependent deviating arguments. Our results are based on the coincidence degree theory. Two examples with singularities of attractive and repulsive type are given.

Keywords. Positive periodic solution; Neutral operator; Time-dependent deviating arguments; Singularities of attractive and repulsive type.
\end{abstract}

2010 Mathematics Subject Classification. 34B16, 34C25.

\section{INTRODUCTION}

Recently, neutral differential equations have received much attention attention. There has been a rapid growth of interest in neutral differential equations which appear in the blood cell production models, control models and population models; see, e.g., [1, 2, 3]. Bai and Xu [1] discussed a two-phase sizestructured population model with infinite states-at-birth and distributed delay in birth process. Recently, topological degree theory, the Krasnoselskii fixed point theorem, and the fixed point theorems in a cone have been employed to investigate the existence of a periodic solutions of neutral nonlinear differential equations; see, e.g., $[4,5,6,7,8,9,10,11,12,13,14]$ and the references therein.

In recent years, some results have been performed on the existence of periodic solutions of neutral differential equations with singularity of repulsive type; see, e.g., [15, 16, 17]. In 2015, Kong, Lu and Liang [15] discussed the following second-order neutral Liénard equation with singularity of repulsive type:

$$
(x(t)-c x(t-\tau))^{\prime \prime}+f(x(t)) x^{\prime}(t)+g(t, x(t-\sigma))=e(t),
$$

${ }^{*}$ Corresponding author.

E-mail address: czb_1982@126.com (Z. Cheng).

Received February 22, 2019; Accepted July 25, 2019.

(C)2019 Journal of Nonlinear Functional Analysis 
where $c$ is a constant with $|c|<1$, and the nonlinear term $g$ repulsive singular at $x=0$, i.e.,

$$
\lim _{x \rightarrow 0^{+}} g_{0}(x)=-\infty, \text { and } \int_{0}^{1} g_{0}(s) d s=-\infty .
$$

They obtained the existence of a positive periodic solution for equation (1.1) by using Mawhin's continuation theorem. In 2017, Xin and Cheng [16] investigated the following second order neutral Rayleigh equation with singularity of repulsive type:

$$
(x(t)-c x(t-\tau))^{\prime \prime}+f\left(t, x^{\prime}\right)+g(t, x(t))=e(t),
$$

where the nonlinear term $g$ satisfies repulsive condition (1.2). Using coincidence degree theory, they obtained the existence of a positive periodic solution for equation (1.3). Recently, Kong and Lu [17] studied a kind of fourth-order Liénard equation with singularity of repulsive type

$$
(x(t)-c x(t-\tau))^{(4)}+f(x(t)) x^{\prime}(t)+g(t, x(t-\sigma(t)))=e(t),
$$

where $c$ is a constant with $|c|<1, f$ is a continuous function, the nonlinear term $g \in C(\mathbb{R} \times(0,+\infty), \mathbb{R})$ is a $T$-periodic function about $t$ and can be singular at $x=0 . e: \mathbb{R} \rightarrow \mathbb{R}$ is a continuous $T$-periodic function and $\int_{0}^{T} e(t) d t=0, \sigma \in C^{1}(\mathbb{R}, \mathbb{R})$ is a $T$-periodic functions and $\sigma^{\prime}(t) \neq 1$. By using the coincidence degree theory, they obtained the following conclusion.

Theorem 1.1. [17] Assume that the following conditions hold:

$\left(F_{1}\right)$ There exist positive constant $d_{1}$ and $d_{2}$ with $d_{1}<d_{2}$ such that $g(t, x)<0$ for all $(t, x) \in[0, T] \times$ $\left(0, d_{1}\right)$, and $g(t, x)>0$ for all $(t, x) \in[0, T] \times\left(d_{2},+\infty\right)$.

$\left(F_{2}\right) \bar{g}(x):=\frac{1}{T} \int_{0}^{T} g(t, x) d t<0$ for all $x \in\left(0, d_{1}\right)$, and $\bar{g}(x)>0$ for all $x \in\left(d_{2},+\infty\right)$.

$\left(F_{3}\right)$ The nonlinear term $g(t, x(t-\sigma(t)))=g_{1}(t, x(t-\sigma(t)))+g_{0}(x(t))$, where $g_{1}:[0, T] \times \mathbb{R} \rightarrow \mathbb{R}$ is a continuo function, and $g_{0}:(0,+\infty) \rightarrow \mathbb{R}$ is a continuous function and satisfying equation (1.2), (i.e. singularity of repulsive type).

$\left(F_{4}\right)$ There exist positive constants $m$ and $n$ such that

$$
g(t, x) \leq m x+n, \text { for all }(t, x) \in[0, T] \times(0,+\infty) .
$$

$\left(F_{5}\right)$ There exist positive constants $\alpha$ and $\beta$ such that

$$
F(x) \leq \alpha x+\beta, \text { for all } x \in(0,+\infty),
$$

where $F(x)=\int_{0}^{x} f(s) d s$.

Then equation (1.4) has at least one positive T-periodic solution if

$$
\frac{(2(1+|c|) a+\alpha|c|) T^{3}}{\left.\pi^{2}|1-| c\right|^{2}}<1 .
$$

In this paper, inspired by the recent results $[15,16,17]$, we consider the existence of a positive $T$ periodic solution for equation (1.4) with singularities of attractive and repulsive type and time-dependent deviating argument, where $c$ is a constant and $|c| \neq 1$. By using the coincidence degree theory, we get the following conclusion.

Theorem 1.2. Assume that the following conditions hold:

$\left(H_{1}\right)$ There exist positive constants $D_{1}$ and $D_{2}$ with $D_{1}<D_{2}$ such that $\bar{g}(x):=\frac{1}{T} \int_{0}^{T} g(t, x) d t>0$ for all $x \in\left(0, D_{1}\right)$, and $\bar{g}(x)<0$ for all $x \in\left(D_{2},+\infty\right)$. 
$\left(H_{2}\right)$ The nonlinear term $g(t, x(t-\sigma(t)))=g_{1}(t, x(t-\sigma(t)))+g_{0}(x(t-\sigma(t)))$, where $g_{1}:[0, T] \times \mathbb{R} \rightarrow$ $\mathbb{R}$ is a continuo function, $g_{0}:(0,+\infty) \rightarrow \mathbb{R}$ is a continuous function.

$\left(H_{3}\right)$ (Singularity of attractive type) $\lim _{x \rightarrow 0^{+}} g_{0}(x)=+\infty$, and $\int_{0}^{1} g_{0}(s) d s=+\infty$.

$\left(\mathrm{H}_{4}\right)$ There exists positive constants $m, n$ such that

$$
-g(t, x) \leq m x+n, \text { for }(t, x) \in \mathbb{R} \times(0,+\infty) .
$$

Then equation (1.4) has at least one positive T-periodic solution if

$$
\frac{(1+|c|)|c|+2 m T^{2}\left(\frac{T}{2 \pi}\right)^{2}}{|1-| c||^{2}}<1 .
$$

Remark 1.3. It is worth mentioning that attractive condition $\left(H_{3}\right)$ contradicts the repulsive condition (1.2). Therefore, the above methods and conditions $\left(F_{1}\right),\left(F_{2}\right),\left(F_{4}\right)$ in $[15,16,17]$ are no long applicable to the existence of a positive periodic solution for equation (1.4) with singularity of attractive type. In this paper, we replace conditions $\left(F_{1}\right),\left(F_{2}\right),\left(F_{4}\right)$ by conditions $\left(H_{1}\right),\left(H_{3}\right),\left(H_{4}\right)$, and we employ another method to discuss this problem.

Remark 1.4. From condition $\left(F_{3}\right)$ in [17], the singular term $g_{0}$ of equation (1.4) has a deviating argument (i.e. $\sigma \equiv 0$ ). The singular term $g_{0}$ of this paper satisfies time-dependent deviating argument (see condition $\left(H_{2}\right)$ ). For example, let

$$
\sigma(t)=\cos ^{2}(t)
$$

It is easy to verify that the work on estimating a lower bounds of a positive periodic solution for equation (1.4) of this paper is more complex than the one in [17].

Remark 1.5. From condition $\left(F_{5}\right)$ in [17], the maximum of the friction term $f(x)$ may be some constant, and the condition $\left(F_{5}\right)$ is hard restrictive. In this paper, the friction term $f(x)$ is a continuous function.

From equation (1.5), we get $|c|<1$, i.e., Theorem 1.2 obtains the existence of a positive periodic solution for equation (1.4) in the case that $|c|<1$. Next, we consider the existence of a positive periodic solution for equation (1.4) in the case that $|c| \neq 1$, (i.e., $|c|<1$ and $|c|>1$ ).

Theorem 1.6. Assume that conditions $\left(H_{1}\right)-\left(H_{4}\right)$ and $\left(F_{5}\right)$ hold. Then equation (1.4) has at least one positive $T$-periodic solution if

$$
\frac{(\alpha|c|+2(1+|c|) m T) T\left(\frac{T}{2 \pi}\right)^{2}}{|1-| c||^{2}}<1 .
$$

Remark 1.7. It is easy to see that the coefficient $c$ of neutral operator satisfies $|c|<1$ in [17] and in Theorem 1.2. However, the coefficient $c$ satisfies $|c|<1$ and $|c|>1$ in Theorem 1.6.

We also investigate the existence of a positive periodic solution for equation (1.4) with singularity of repulsive type. From Theorems 1.2 and 1.6, we get the following conclusions.

Theorem 1.8. Assume that conditions $\left(F_{2}\right),\left(F_{4}\right),\left(H_{3}\right)$ and equations (1.2), (1.5) hold. Then equation (1.4) has at least one positive T-periodic solution.

Theorem 1.9. Assume that conditions $\left(F_{2}\right),\left(F_{4}\right),\left(F_{5}\right),\left(H_{3}\right)$ and equations (1.2), (1.6) hold. Then equation (1.4) has at least one positive T-periodic solution.

Remark 1.10. Obviously, Theorem 1.9 implies Theorem 1.1, and condition $\left(H_{3}\right)$ is relatively weaker than condition $\left(F_{3}\right)$. 


\section{PRELIMINARIES}

First, we recall the coincidence degree theory.

Lemma 2.1. [18] Let $X$ and $Y$ be two Banach spaces, and let $L: D(L) \subset X \rightarrow Y$ be a Fredholm operator with index zero. Let $\Omega \subset X$ be an open bounded set, and let $N: \bar{\Omega} \rightarrow Y$ be L-compact on $\bar{\Omega}$. Assume that the following conditions hold:

(1) $L x \neq \lambda N x, \forall x \in \partial \Omega \cap D(L), \lambda \in(0,1)$;

(2) $N x \notin \operatorname{Im} L, \forall x \in \partial \Omega \cap \operatorname{Ker} L$;

(3) $\operatorname{deg}\left\{\left.Q N\right|_{\text {Ker } L}, \Omega \cap \operatorname{Ker} L, 0\right\} \neq 0$.

Then the equation $L x=N x$ has a solution in $\bar{\Omega} \cap D(L)$.

Lemma 2.2. [19] If $|c| \neq 1$, then $(A x)(t):=x(t)-c x(t-\tau)$ has continuous bounded inverse on $C_{T}:=$ $\{x \in C(\mathbb{R}, \mathbb{R}) \mid x(t+T)-x(t) \equiv 0\}$ and

$$
\int_{0}^{T}\left|\left(A^{-1} x\right)(t)\right|^{2} d t \leq \frac{1}{|1-| c||^{2}} \int_{0}^{T}|x(t)|^{2} d t, \forall x \in C_{T} .
$$

Now, set

$$
X:=\left\{x \in C^{1}\left(\mathbb{R}, \mathbb{R}^{2}\right): x(t+T)-x(t) \equiv 0\right\}
$$

with the norm $\|x\|_{\infty}:=\max \left\{\|x\|,\left\|x^{\prime}\right\|\right\}$;

$$
Y:=\{x \in C(\mathbb{R}, \mathbb{R}): x(t+T)-x(t) \equiv 0\}
$$

with the norm $\|x\|:=\max \|x\|$. Clearly, both $X$ and $Y$ are Banach spaces. Meanwhile, we define

$$
L: D(L):=\left\{x \in C^{4}\left(\mathbb{R}, \mathbb{R}^{2}\right): x(t+T)-x(t) \equiv 0, \text { for } t \in \mathbb{R}\right\} \subset X \rightarrow Y
$$

by

$$
(L x)(t)=(A x)^{(4)}(t)
$$

and $N: X \rightarrow Y$ by

$$
(N x)(t)=-f(x(t)) x^{\prime}(t)-g(t, x(t-\sigma(t)))+e(t) .
$$

Then equation (1.4) can be converted to the abstract equation $L x=N x$. From the definition of $L$, one can easily see that

$$
\operatorname{Ker} L \cong \mathbb{R}, \operatorname{Im} L=\left\{y \in Y: \int_{0}^{T} y(s) d s=0\right\} .
$$

So $L$ is a Fredholm operator with index zero. Let $P: X \rightarrow \operatorname{Ker} L$ and $Q: Y \rightarrow \operatorname{Im} Q \subset \mathbb{R}$ be defined by

$$
P x:=(A x)(0) ; \text { and } Q y:=\frac{1}{T} \int_{0}^{T} y(s) d s .
$$

Then $\operatorname{Im} P=\operatorname{Ker} L$, and $\operatorname{Ker} Q=\operatorname{Im} L$. Denote $L_{p}=\left.L\right|_{D(L) \cap K e r P}$ and let $L_{P}^{-1}: \operatorname{Im} L \rightarrow D(L)$ be inverse of $L_{p}$. Then

$$
\begin{aligned}
& {\left[L_{p}^{-1} y\right](t)=\left(A^{-1} G y\right)(t),} \\
& (G y)(t)=\sum_{i=1}^{3} \frac{1}{3 !} a_{i} t^{i}+\frac{1}{3 !} \int_{0}^{t}(t-s)^{3} y(s) d s,
\end{aligned}
$$


where $a_{i}:=(A x)^{(i)}(0), i=1,2,3$ are defined as follows

$$
\left(\begin{array}{c}
(A x)^{\prime \prime \prime}(0) \\
(A x)^{\prime \prime}(0) \\
(A x)^{\prime}(0)
\end{array}\right)\left(\begin{array}{ccc}
1 & 0 & 0 \\
\frac{T}{2} & 1 & 0 \\
\frac{T^{2}}{3} & \frac{T}{2} & 1
\end{array}\right)=\left(\begin{array}{c}
-\frac{1}{T} \int_{0}^{T}(T-s) y(s) d s \\
-\frac{1}{2 ! T} \int_{0}^{T}(T-s)^{2} y(s) d s \\
-\frac{1}{3 ! T} \int_{0}^{T}(T-s)^{3} y(s) d s
\end{array}\right) .
$$

From equations (2.1) and (2.2), we get that $N$ is $L$-compact on $\bar{\Omega}$.

\section{Proof of Theorem 1.2}

Consider the equation

$$
L x=\lambda N x, \lambda \in(0,1) .
$$

Set $\Omega_{1}=\{x: L x=\lambda N x, \lambda \in(0,1)\}$. If $x(t) \in \Omega_{1}$, then

$$
((A x)(t))^{(4)}+\lambda f(x(t)) x^{\prime}(t)+\lambda g(t, x(t-\sigma(t)))=\lambda e(t) .
$$

Integrating both side of equation (3.1) over $[0, T]$, we have

$$
\int_{0}^{T} g(t, x(t-\sigma(t))) d t=0
$$

Then by condition $\left(H_{1}\right)$, there exist positive constants $D_{1}, D_{2}$ and $\eta \in(0, T)$ such that

$$
D_{1} \leq x(\eta-\sigma(\eta)) \leq D_{2}
$$

Setting $\xi=\eta-\sigma(\eta) \in(0, T)$, we have

$$
D_{1} \leq x(\xi) \leq D_{2}
$$

It follows that

$$
\|x\|=\max _{t \in[0, T]}|x(t)|=\max _{t \in[0, T]}\left|x(\xi)+\int_{\xi}^{t} x^{\prime}(s) d s\right| \leq D_{2}+\int_{0}^{T}\left|x^{\prime}(s)\right| d s .
$$

Multiplying both sides of equation (3.1) by $x(t)$ and integrating over the interval $[0, T]$, we get

$$
\int_{0}^{T}((A x)(t))^{(4)} x(t) d t=-\lambda \int_{0}^{T} f(x(t)) x^{\prime}(t) x(t) d t-\lambda \int_{0}^{T} g(t, x(t-\sigma(t))) x(t) d t+\lambda \int_{0}^{T} e(t) x(t) d t .
$$

Substituting $\int_{0}^{T}((A x)(t))^{(4)} x(t) d t=\int_{0}^{T}(A x)^{\prime \prime}(t) x^{\prime \prime}(t) d t, \int_{0}^{T} f(x(t)) x^{\prime}(t) x(t) d t=0$ into equation (3.6), we see that

$$
\int_{0}^{T}(A x)^{\prime \prime}(t) x^{\prime \prime}(t) d t=-\lambda \int_{0}^{T} g(t, x(t-\sigma(t))) x(t) d t+\lambda \int_{0}^{T} e(t) x(t) d t .
$$

Furthermore,

$$
\begin{aligned}
\int_{0}^{T}(A x)^{\prime \prime}(t) x^{\prime \prime}(t) d t & =\int_{0}^{T}(A x)^{\prime \prime}(t)\left((A x)^{\prime \prime}(t)+c x^{\prime \prime}(t-\tau)\right) d t \\
& =\int_{0}^{T}\left|(A x)^{\prime \prime}(t)\right|^{2} d t+c \int_{0}^{T} x^{\prime \prime}(t-\tau)\left(A x^{\prime \prime}\right)(t) d t,
\end{aligned}
$$

since $(A x)^{\prime \prime}(t)=\left(A x^{\prime \prime}\right)(t)$. Substituting equation (3.8) into equation (3.7), we deduce

$$
\begin{aligned}
\int_{0}^{T}\left|(A x)^{\prime \prime}(t)\right|^{2} d t & =-c \int_{0}^{T} x^{\prime \prime}(t-\tau)\left(A x^{\prime \prime}\right)(t) d t-\lambda \int_{0}^{T} g(t, x(t-\sigma(t))) x(t) d t+\lambda \int_{0}^{T} e(t) x(t) d t \\
& =|c| \int_{0}^{T}\left|x^{\prime \prime}(t-\tau)\right|\left|\left(A x^{\prime \prime}\right)(t)\right| d t+\|x\| \int_{0}^{T}|g(t, x(t-\sigma(t)))| d t+\int_{0}^{T}|e(t)||x(t)| d t .
\end{aligned}
$$


Applying the Hölder inequality and the Minkouski inequality, it is clear that

$$
\begin{aligned}
& \int_{0}^{T}\left|x^{\prime \prime}(t-\tau)\right|\left|\left(A x^{\prime \prime}\right)(t)\right| d t \\
\leq & \left(\int_{0}^{T} \mid x^{\prime \prime}(t-\tau) d t\right)^{\frac{1}{2}}\left(\int_{0}^{T}\left|\left(A x^{\prime \prime}\right)(t)\right|^{2} d t\right)^{\frac{1}{2}} \\
\leq & \left(\int_{0}^{T}\left|x^{\prime \prime}(t)\right|^{2} d t\right)^{\frac{1}{2}}\left(\left(\int_{0}^{T}\left|x^{\prime \prime}(t)\right|^{2} d t\right)^{\frac{1}{2}}+|c|\left(\int_{0}^{T}\left|x^{\prime \prime}(t-\tau)\right|^{2} d t\right)^{\frac{1}{2}}\right) \\
\leq & \left(\int_{0}^{T}\left|x^{\prime \prime}(t)\right|^{2} d t\right)^{\frac{1}{2}}\left(\left(\int_{0}^{T}\left|x^{\prime \prime}(t)\right|^{2} d t\right)^{\frac{1}{2}}+|c|\left(\int_{0}^{T}\left|x^{\prime \prime}(t)\right|^{2} d t\right)^{\frac{1}{2}}\right) \\
= & (1+|c|) \int_{0}^{T}\left|x^{\prime \prime}(t)\right|^{2} d t
\end{aligned}
$$

since $\int_{0}^{T}\left|x^{\prime \prime}(t-\tau)\right|^{2} d t=\int_{0}^{T}\left|x^{\prime \prime}(t)\right|^{2} d t$. On the other hand, form $\left(H_{2}\right)$ and equation (3.2), we have

$$
\begin{aligned}
& \int_{0}^{T}|g(t, x(t-\sigma(t)))| d t \\
= & \int_{g(t, x(t-\sigma(t)) \geq 0} g^{+}(t, x(t-\sigma(t))) d t-\int_{g(t, x(t-\sigma(t)) \leq 0} g^{-}(t, x(t-\sigma(t))) d t \\
= & -2 \int_{g(t, x(t-\sigma(t)) \leq 0} g^{-}(t, x(t-\sigma(t))) d t \\
\leq & 2 m \int_{0}^{T} x(t-\sigma(t)) d t+2 n T \\
\leq & 2 m\|x\| T+2 n T,
\end{aligned}
$$

where $g^{+}:=\max \{g(t, x), 0\}, g^{-}:=\min \{g(t, x), 0\}$. Substituting equations (3.10) and (3.11) into equation (3.9), we get from the Hölder inequality that

$$
\begin{aligned}
& \int_{0}^{T}\left|(A x)^{\prime \prime}(t)\right|^{2} d t \\
\leq & (1+|c|)|c| \int_{0}^{T}\left|x^{\prime \prime}(t)\right|^{2} d t+2 m T\|x\|^{2}+2 n T\|x\|+\|e\|\|x\| T \\
\leq & (1+|c|)|c| \int_{0}^{T}\left|x^{\prime \prime}(t)\right|^{2} d t+2 m T\left(D_{2}+\int_{0}^{T}\left|x^{\prime}(t)\right| d t\right)^{2}+\left(D_{2}+\int_{0}^{T}\left|x^{\prime}(t)\right| d t\right) \\
& (n T+\|e\| T) \\
\leq & (1+|c|)|c| \int_{0}^{T}\left|x^{\prime \prime}(t)\right|^{2} d t+2 m T^{2} \int_{0}^{T}\left|x^{\prime}(t)\right|^{2} d t+E_{1}\left(\int_{0}^{T}\left|x^{\prime}(t)\right|^{2} d t\right)^{\frac{1}{2}}+E_{2},
\end{aligned}
$$

where

$$
E_{1}:=4 m T D_{2}+(2 n T+\|e\| T) T^{\frac{1}{2}}
$$

and

$$
E_{2}:=2 m T D_{2}^{2}+D_{2}(2 n T+\|e\| T) .
$$


From the Wirtinger inequality (see [20]), we have

$$
\begin{aligned}
\int_{0}^{T}\left|(A x)^{\prime \prime}(t)\right|^{2} d t \leq & (1+|c|)|c| \int_{0}^{T}\left|x^{\prime \prime}(t)\right|^{2} d t+2 m T^{2}\left(\frac{T}{2 \pi}\right)^{2} \int_{0}^{T}\left|x^{\prime \prime}(t)\right|^{2} d t \\
& +E_{1}\left(\frac{T}{2 \pi}\right)\left(\int_{0}^{T}\left|x^{\prime \prime}(t)\right|^{2} d t\right)^{\frac{1}{2}}+E_{2} .
\end{aligned}
$$

Since $(A x)^{\prime \prime}(t)=\left(A x^{\prime \prime}\right)(t)$. Form Lemma 2.2 and equation (3.14), we obtain

$$
\begin{aligned}
& \int_{0}^{T}\left|x^{\prime \prime}(t)\right|^{2} d t \\
= & \int_{0}^{T}\left|\left(A^{-1} A x^{\prime \prime}\right)(t)\right|^{2} d t \\
\leq & \frac{1}{|1-| c||^{2}} \int_{0}^{T}\left|(A x)^{\prime \prime}(t)\right|^{2} d t \\
\leq & \frac{1}{|1-| c||^{2}}\left(\left((1+|c|)|c|+2 m T^{2}\left(\frac{T}{2 \pi}\right)^{2}\right) \int_{0}^{T}\left|x^{\prime \prime}(t)\right|^{2} d t\right. \\
& \left.+E_{1}\left(\frac{T}{2 \pi}\right)\left(\int_{0}^{T}\left|x^{\prime \prime}(t)\right|^{2} d t\right)^{\frac{1}{2}}+E_{2}\right) \\
= & \frac{(1+|c|)|c|+2 m T^{2}\left(\frac{T}{2 \pi}\right)^{2}}{|1-| c||^{2}} \int_{0}^{T}\left|x^{\prime \prime}(t)\right|^{2} d t+\frac{E_{1} \frac{T}{2 \pi}}{|1-| c||^{2}}\left(\int_{0}^{T}\left|x^{\prime \prime}(t)\right|^{2} d t\right)^{\frac{1}{2}}+\frac{E_{2}}{|1-| c||^{2}} .
\end{aligned}
$$

Since

$$
\frac{(1+|c|)|c|+2 m T^{2}\left(\frac{T}{2 \pi}\right)^{2}}{|1-| c||^{2}}<1,
$$

we see that there exists a positive constant $M_{1}^{\prime}$ such that

$$
\int_{0}^{T}\left|x^{\prime \prime}(t)\right|^{2} d t \leq M_{1}^{\prime}
$$

From equation (3.6) and the Wirtinger inequality, we have

$$
\begin{aligned}
\|x\| & \leq D_{2}+\int_{0}^{T}\left|x^{\prime}(t)\right| d t \\
& \leq D_{2}+\sqrt{T}\left(\frac{T}{2 \pi}\right)\left(\int_{0}^{T}\left|x^{\prime \prime}(t)\right|^{2} d t\right)^{\frac{1}{2}} \\
& \leq D_{2}+\sqrt{T}\left(\frac{T}{2 \pi}\right)\left(M_{1}^{\prime}\right)^{\frac{1}{2}}:=M_{1} .
\end{aligned}
$$

In view of $x(0)=x(T)$, we know that there exists a point $t_{2} \in(0, T)$ such that $x^{\prime}\left(t_{2}\right)=0$, we get from equation (3.5) and the Hölder inequality that

$$
\begin{aligned}
\left\|x^{\prime}\right\| & \leq \int_{0}^{T}\left|x^{\prime \prime}(t)\right| d t \\
& \leq \sqrt{T}\left(\int_{0}^{T}\left|x^{\prime \prime}(t)\right|^{2} d t\right)^{\frac{1}{2}} \\
& \leq \sqrt{T}\left(M_{1}^{\prime}\right)^{\frac{1}{2}}:=M_{2} .
\end{aligned}
$$


It follows from equation (3.1) and $g(t, x)=g_{0}(x)+g_{1}(t, x)$ that

$$
((A x)(t))^{(4)}+\lambda f(x(t)) x^{\prime}(t)+\lambda\left(g_{0}(x(t-\sigma(t)))+g_{1}(t, x(t-\sigma(t)))=\lambda e(t) .\right.
$$

Let $\eta \in[0, T]$ be as in equation (3.3), for any $\eta \leq t \leq T$. From equations (3.3) and (3.15), we have

$$
x(\eta-\sigma(\eta)) \geq D_{1} .
$$

Next, we show that, for any $t \in[\eta, T]$, there exists a constant $D_{3} \in\left(0, D_{1}\right)$ such that each positive $T$ periodic solution of equation (1.4) satisfies

$$
x(t-\sigma(t))>D_{3} .
$$

In fact, multiplying both sides of equation (3.16) by $x^{\prime}(t-\sigma(t))\left(1-\sigma^{\prime}(t)\right)$ and integrating on $[\eta, t]$ yield that

$$
\begin{aligned}
\lambda \int_{x(\eta-\sigma(\eta))}^{x(t-\sigma(t))} g_{0}(u) d u= & \lambda \int_{\eta}^{t} g_{0}(x(s-\sigma(s))) x^{\prime}(s-\sigma(s))\left(1-\sigma^{\prime}(s)\right) d s \\
= & -\int_{\eta}^{t}((A x)(s))^{(4)} x^{\prime}(s-\sigma(s))\left(1-\sigma^{\prime}(s)\right) d s \\
& -\lambda \int_{\eta}^{t} f(x(s)) x^{\prime}(s) x^{\prime}(s-\sigma(s))\left(1-\sigma^{\prime}(s)\right) d s \\
& -\lambda \int_{\eta}^{t} g_{1}(s, x(s-\sigma(s))) x^{\prime}(s-\sigma(s))\left(1-\sigma^{\prime}(s)\right) d s \\
& +\lambda \int_{\eta}^{t} e(s) x^{\prime}(s-\sigma(s))\left(1-\sigma^{\prime}(s)\right) d s .
\end{aligned}
$$

Furthermore, we have

$$
\begin{aligned}
\lambda\left|\int_{x(\eta-\sigma(\eta))}^{x(t-\sigma(t))} g_{0}(v) d v\right| \leq & \left|\int_{\eta}^{t}((A x)(s))^{(4)} x^{\prime}(s-\sigma(s))\left(1-\sigma^{\prime}(s)\right) d s\right| \\
& +\lambda\left|\int_{\eta}^{t} f(x(s)) x^{\prime}(s) x^{\prime}(s-\sigma(s))\left(1-\sigma^{\prime}(s)\right) d s\right| \\
& +\lambda\left|\int_{\eta}^{t} g_{1}(s, x(s-\sigma(s))) x^{\prime}(s-\sigma(s))\left(1-\sigma^{\prime}(s)\right) d s\right| \\
& +\lambda\left|\int_{\eta}^{t} e(s) x^{\prime}(s-\sigma(s))\left(1-\sigma^{\prime}(s)\right)\right|
\end{aligned}
$$

By using equations (3.1), (3.14) and (3.15), we have

$$
\begin{aligned}
\left|\int_{\eta}^{t}((A x)(s))^{(4)} x^{\prime}(s-\sigma(s))\left(1-\sigma^{\prime}(s)\right) d s\right| & \int_{\eta}^{t}\left|((A x)(s))^{(4)}\right|\left|x^{\prime}(s-\sigma(s)) \| 1-\sigma^{\prime}(s)\right| d s \\
\leq & \sigma^{1}\left\|x^{\prime}\right\| \int_{0}^{T}\left|((A x)(s))^{(4)}\right| d t \\
\leq & \lambda \sigma^{1} M_{2}\left(\int_{0}^{T}\left|f(x(s)) \| x^{\prime}(s)\right| d t\right. \\
& \left.+\int_{0}^{T}|g(s, x(s-\sigma(s)))| d t+\int_{0}^{T}|e(s)| d t\right) \\
\leq & \lambda \sigma^{1} M_{2}\left(|f|_{M_{1}} M_{2} T+2 m T M_{1}+2 n T+T\|e\|\right),
\end{aligned}
$$


where $|f|_{M_{1}}:=\max _{0<x \leq M_{1}}|f(x)|$ and $\sigma^{1}:=\max _{t \in[0, T]}\left|1-\sigma^{\prime}(t)\right|$. Besides, we have

$$
\begin{aligned}
& \left|\int_{\eta}^{t} f(x(s)) x^{\prime}(s) x^{\prime}(s-\sigma(s))\left(1-\sigma^{\prime}(s)\right) d s\right| \leq \sigma^{1} M_{2}^{2} \int_{0}^{T}|f(x(u(s)))| d s \leq\left(1-\sigma_{0}^{1}\right) M_{2}^{2} T|f|_{M_{1}} . \\
& \left|\int_{\eta}^{t} g_{1}(s, x(s-\sigma(s))) x^{\prime}(s-\sigma(s))\left(1-\sigma^{\prime}(s)\right) d s\right| \leq \sigma^{1} M_{2} \sqrt{T}\left\|g_{M_{1}}\right\|_{2} . \\
& \left|\int_{\eta}^{t} e(s) x^{\prime}(s-\sigma(s))\left(1-\sigma^{\prime}(s)\right) d s\right| \leq \sigma^{1} M_{2} T\|e\| .
\end{aligned}
$$

where $g_{M_{1}}=\max _{0<x \leq M_{1}}\left|g_{1}(t, x)\right| \in L^{2}(0, T)$. From those inequalities and equation (3.17), we derive

$$
\left|\int_{x(\eta-\sigma(\eta))}^{x(t-\sigma(t))} g_{0}(u) d u\right| \leq \sigma^{1}\left(2 M_{2} T\left(M_{2}|f|_{M_{1}}+m M_{1}+n+\frac{1}{2 \sqrt{T}}\left\|g_{M_{1}}\right\|_{2}+\|e\|\right)\right):=M_{3} .
$$

In view of attractive condition $\left(H_{4}\right)$ and $x(\eta-\sigma(\eta)) \geq D_{1}$, there exists $D_{3} \in\left(0, D_{1}\right)$ such that

$$
\left|\int_{D_{1}}^{D_{3}} g_{0}(v) d v\right|>M_{3}
$$

Thus, if there exists a point $\delta \in[0, T]$ such that $x(\eta-\sigma(\eta)) \leq D_{3}$, then

$$
\left|\int_{x(\eta-\sigma(\eta))}^{x(\delta-\sigma(\delta))} g_{0}(v) d v\right| \geq\left|\int_{D_{1}}^{D_{3}} g_{0}(v) d v\right|>M_{3}
$$

which contradicts equation (3.18). Therefore, we can obtain that

$$
x(t-\sigma(t)) \geq D_{3}, \forall t \in[0, T] .
$$

Letting $u=t-\sigma(t)$, we have

$$
x(u) \geq D_{3}, \forall u \in[\sigma(0), T-\sigma(T)],
$$

i.e., $x(u) \geq D_{3}, \forall u \in[0, T]$, since $\sigma(0)=\sigma(T)$. Define

$$
\Omega=\left\{x \in C_{T}^{1}(\mathbb{R}, \mathbb{R}) \mid E_{1} \leq x \leq E_{2},\left\|x^{\prime}\right\| \leq E_{3}, \forall t \in[0, T]\right\},
$$

where $0<E_{1}<\min \left(D_{3}, D_{1}\right), E_{2}>\max \left(M_{1}, D_{2}\right), E_{3}>M_{2}$. Let $\Omega_{2}=\{x: x \in \partial \Omega \cap \operatorname{Ker} L\}$. Then $\forall x \in \partial \Omega \cap \operatorname{Ker} L$,

$$
Q N x=-\frac{1}{T} \int_{0}^{T} g(t, x(t-\sigma(t) d t
$$

since $\int_{0}^{T} f(x(t)) x^{\prime}(t) d t=0$ and $\int_{0}^{T} e(t) d t=0$. If $Q N x=0$, then $x(t)=E_{1}$ or $x(t)=E_{2}$. But if $x(t)=E_{2}$, we know

$$
\int_{0}^{T} g\left(t, E_{2}\right) d t=0
$$

From condition $\left(H_{1}\right)$, we have $x(t) \leq E_{2} \leq D_{2}$, which yields a contradiction. Similarly if $x_{1}=E_{1}$, we also have $Q N x \neq 0$, i.e., $\forall x \in \partial \Omega \cap \operatorname{Ker} L, x \notin \operatorname{Im} L$. So assumptions (1) and (2) of Lemma 2.1 are both satisfied. Let

$$
H(\mu, x)=-\mu x-\frac{1-\mu}{T} \int_{0}^{T} g(t, x(t-\sigma(t)) d t,(\mu, x) \in[0,1] \times \Omega .
$$

From condition $\left(H_{1}\right)$, it is obvious that $x H(\mu, x) \neq 0, \forall(\mu, x) \in[0,1] \times(\partial \Omega \cap \operatorname{Ker} L)$. Hence

$$
\begin{aligned}
\operatorname{deg}\left\{\left.Q N\right|_{\text {Ker } L}, \Omega \cap \operatorname{Ker} L, 0\right\} & =\operatorname{deg}\{H(0, x), \Omega \cap \operatorname{Ker} L, 0\} \\
& =\operatorname{deg}\{H(1, x), \Omega \cap \operatorname{Ker} L, 0\} \neq 0 .
\end{aligned}
$$


So assumption (3) of Lemma 2.1 is satisfied. Applying Lemma 2.1, we conclude that equation $L x=N x$ has a solution $x$ on $\bar{\Omega} \cap D(L)$, i.e., equation (1.4) has a positive $T$-periodic solution $x(t)$. This completes the proof.

\section{Proof of Theorem 1.6}

We follow the same strategy and notation as in the proof of Theorem 1.2. We consider that there exists a positive constant $M_{1}^{\prime}$ such that

$$
\int_{0}^{T}\left|x^{\prime \prime}(t)\right|^{2} d t \leq M_{1}^{\prime}
$$

In fact, multiplying both side of equation $(3.1)$ by $(A x)(t)$ and integrating over $[0, T]$, we deduce

$$
\begin{aligned}
\int_{0}^{T}(A x)^{(4)}(t)(A x)(t) d t= & -\lambda \int_{0}^{T} f(x(t)) x^{\prime}(t)(A x)(t) d t-\lambda \int_{0}^{T} g(t, x(t-\sigma(t)))(A x)(t) d t \\
& +\lambda \int_{0}^{T} e(t)(A x)(t) d t
\end{aligned}
$$

Note that

$$
\begin{aligned}
\int_{0}^{T} f(x(t)) x^{\prime}(t)(A x)(t) d t & =\int_{0}^{T} f(x(t)) x^{\prime}(t)(x(t)-c x(t-\tau)) d t \\
& =-c \int_{0}^{T} f(x(t)) x^{\prime}(t) x(t-\tau) d t \\
& =c \int_{0}^{T} F(x(t)) x^{\prime}(t-\tau) d t
\end{aligned}
$$

where $F(x)=\int_{0}^{x} f(s) d s$. Substituting $\int_{0}^{T}(A x)^{(4)}(t)(A x)(t) d t=\int_{0}^{T}\left|(A x)^{\prime \prime}(t)\right|^{2} d t$ and (4.3) into (4.2), we see from condition $\left(F_{5}\right)$ and equation (3.11) that

$$
\begin{aligned}
\int_{0}^{T}\left|(A x)^{\prime \prime}(t)\right|^{2} d t \leq & |c| \int_{0}^{T}|F(x(t))|\left|x^{\prime}(t-\tau)\right| d t+(1+|c|)\|x\| \int_{0}^{T}|g(t, x(t-\sigma(t)))| d t \\
& +(1+|c|)\|x\| \int_{0}^{T}|e(t)| d t \\
\leq & \alpha|c| \int_{0}^{T}\left|x(t) \| x^{\prime}(t-\tau)\right| d t+\beta|c| \int_{0}^{T}\left|x^{\prime}(t-\tau)\right| d t \\
& +(1+|c|)\|x\|(2 m T\|x\|+2 n T)+(1+|c|) T\|x\|\|e\| \\
\leq & \alpha|c|\|x\| \int_{0}^{T}\left|x^{\prime}(t)\right| d t+\beta|c| \int_{0}^{T}\left|x^{\prime}(t)\right| d t+(1+|c|) 2 m T\|x\|^{2}+N_{1}\|x\|,
\end{aligned}
$$

where

$$
N_{1}:=(1+|c|)(2 n T+\|e\| T)
$$

and

$$
\int_{0}^{T}\left|x^{\prime}(t-\tau)\right| d t=\int_{0}^{T}\left|x^{\prime}(t)\right| d t .
$$


Substituting (3.5) into (4.4), and applying the Hölder inequality and the Wirtinger inequality, we obtain

$$
\begin{aligned}
\int_{0}^{T}\left|(A x)^{\prime \prime}(t)\right|^{2} d t \leq & \alpha|c|\left(D_{2}+\int_{0}^{T}\left|x^{\prime}(t)\right| d t\right) \int_{0}^{T}\left|x^{\prime}(t)\right| d t+\beta|c| \int_{0}^{T}\left|x^{\prime}(t)\right| d t \\
& +(1+|c|) 2 m T\left(D_{2}+\int_{0}^{T}\left|x^{\prime}(t)\right| d t\right)^{2}+N_{1}\left(D_{2}+\int_{0}^{T}\left|x^{\prime}(t)\right| d t\right) \\
= & (\alpha|c|+(1+|c|) 2 m T)\left(\int_{0}^{T}\left|x^{\prime}(t)\right| d t\right)^{2}+\left(\alpha|c| D_{2}+|c| \beta+(1+|c|) 4 m T D_{2}+N_{1}\right) \\
& \cdot \int_{0}^{T}\left|x^{\prime}(t)\right| d t+(1+|c|) 2 m T D_{2}+N_{1} D_{2} \\
\leq & (\alpha|c|+(1+|c|) 2 m T) T \int_{0}^{T}\left|x^{\prime}(t)\right|^{2} d t+N_{2} T^{\frac{1}{2}}\left(\int_{0}^{T}\left|x^{\prime}(t)\right|^{2} d t\right)^{\frac{1}{2}}+N_{3} \\
\leq & (\alpha|c|+(1+|c|) 2 m T) T\left(\frac{T}{2 \pi}\right)^{2} \int_{0}^{T}\left|x^{\prime \prime}(t)\right|^{2} d t+N_{2} T^{\frac{1}{2}}\left(\frac{T}{2 \pi}\right)\left(\int_{0}^{T}\left|x^{\prime \prime}(t)\right|^{2} d t\right)^{\frac{1}{2}}+N_{3},
\end{aligned}
$$

where

$$
N_{2}:=\alpha|c| D_{2}+|c| \beta+(1+|c|) 4 m T D_{2}+N_{1}
$$

and

$$
N_{3}:=(1+|c|) 2 m T D_{2}+N_{1} D_{2} .
$$

From (3.15), (4.5) and Lemma 2.2, we get

$$
\begin{aligned}
\int_{0}^{T}\left|x^{\prime \prime}(t)\right|^{2} d t \leq & \frac{(\alpha|c|+(1+|c|) 2 m T) T\left(\frac{T}{2 \pi}\right)^{2}}{|1-| c||^{2}} \int_{0}^{T}\left|x^{\prime \prime}(t)\right|^{2} d t \\
& +\frac{N_{2} T\left(\frac{T}{2 \pi}\right)}{|1-| c||^{2}}\left(\int_{0}^{T}\left|x^{\prime \prime}(t)\right| d t\right)^{\frac{1}{2}}+\frac{N_{3}}{|1-| c||^{2}} .
\end{aligned}
$$

From equation (1.6), it is easy to verify that equation (4.1) hold. The proof left is as same as Theorem 1.2. This completes the proof.

\section{PROOF OF TheOREMS 1.8 AND 1.9}

Since the proof is similar, we only give the proof to Theorem 1.8. With the same method, we can prove Theorem 1.9. We follow the same strategy and notation as in the proof of Theorem 1.2. We only consider $\int_{0}^{T}|g(t, x(t-\sigma(t)))| d t$. From equations (3.11) and condition $\left(F_{4}\right)$, we have

$$
\begin{aligned}
\int_{0}^{T}|g(t, x(t-\sigma(t)))| d t & =\int_{g(t, x)) \geq 0} g(t, x(t-\sigma(t))) d t-\int_{g(t, x) \leq 0} g(t, x(t-\sigma(t))) d t \\
& =2 \int_{g(t, x) \leq 0} g^{+}(t, x(t-\sigma(t))) d t \\
& \leq 2 m \int_{0}^{T} x(t-\sigma(t)) d t+2 n \\
& \leq 2 m T\|x\|+2 n,
\end{aligned}
$$

The proof left is as same as Theorem 1.2. 


\section{EXAMPLES}

In this section, we present two examples to illustrate the existence results involved in our Theorems.

Example 6.1. Consider the fourth-order differential equation with singularity of attractive type and timedependent deviating arguments:

$$
\left(x(t)-\frac{1}{10} x(t-\tau)\right)^{(4)}+f(x(t)) x^{\prime}(t)-\frac{1}{4 \pi^{2}}(\sin 2 t+3) x\left(t-\cos ^{2} t\right)+\frac{1}{x^{\kappa}\left(t-\cos ^{2} t\right)}=\cos 2 t
$$

where $\kappa \geq 1, f$ is a continuous function, $\tau$ is a constant and $0 \leq \tau<T$.

It is clear that $T=\pi, c=\frac{1}{10}, \sigma(t)=\cos ^{2} t$,

$$
g(t, x)=-\frac{1}{4 \pi^{2}}(\sin 2 t+3) x\left(t-\cos ^{2} t\right)+\frac{1}{x^{\kappa}\left(t-\cos ^{2} t\right)}
$$

and $m=\frac{1}{\pi^{2}}$. It is obvious that $\left(H_{1}\right)-\left(H_{4}\right)$ hold. Now we consider

$$
\begin{aligned}
& \frac{(1+|c|)|c|+2 m T^{2}\left(\frac{T}{2 \pi}\right)^{2}}{\left.|1-| c\right|^{2}} \\
= & \frac{\left(1+\frac{1}{10}\right) \times \frac{1}{10}+2 \times \frac{1}{4 \pi^{2}} \times \pi^{2} \times\left(\frac{\pi}{2 \pi}\right)^{2}}{\left|1-\frac{1}{10}\right|^{2}} \\
= & \frac{61}{81}<1 .
\end{aligned}
$$

From Theorem 1.2, we know equation (6.1) has at least one positive $\pi$-periodic solution.

Example 6.2. Consider the fourth-order equation with singularity of repulsive type:

$$
(x(t)+100 x(t-\tau))^{(4)}+\left(e^{\cos x}+\frac{1}{2}\right) x^{\prime}(t)+\frac{1}{10 \pi}(\cos t+4) x\left(t-\frac{1}{2} \sin t\right)+\frac{1}{x^{\rho}\left(t-\frac{1}{2} \sin t\right)}=\frac{1}{4} \sin t,
$$

where $\rho \geq 1, \tau$ is a constant and $0 \leq \tau<T$. It is clear that $T=2 \pi, c=-100, \sigma(t)=\frac{1}{2} \sin t, g(t, x)=$ $\frac{1}{10 \pi}(\cos t+4) x+\frac{1}{x^{\rho}}, m=\frac{1}{2 \pi}, f(x)=e^{\cos x}+\frac{1}{2}, \alpha=\frac{1}{2}$. It is obvious that $\left(F_{2}\right),\left(F_{4}\right),\left(F_{5}\right)$ and $\left(H_{3}\right)$, equation (1.2) hold. Now we consider

$$
\begin{aligned}
& \frac{(\alpha|c|+2(1+|c|) m T) T\left(\frac{T}{2 \pi}\right)^{2}}{|1-| c||^{2}} \\
= & \frac{\frac{1}{2} \times 100+2 \times 101 \times \frac{1}{2 \pi} \times 2 \pi}{99^{2}} \\
= & \frac{504 \pi}{9801}<1 .
\end{aligned}
$$

Therefore, applying Theorem 1.8, we know equation (6.2) has at least one positive $2 \pi$-periodic solution.

\section{CONCLUSION}

In this paper, we discussed existence of a periodic solution for the fourth-order neutral differential equation with singularities of attractive and repulsive type and time-dependent deviating arguments. Due to the attractive condition is contradicted with the repulsive condition, the methods of $[15,16,17]$ are no longer applicable to prove the existence of periodic solutions for the equation (1.4) with singularity of 
attractive singularity. We obtained the existence of a positive periodic solution for equation (1.4) in the case that $|c| \neq 1$ by using the coincidence degree theory.

\section{REFERENCES}

[1] M. Bai, S. Xu, On a two-phase size-structured population model with infinite states-at-birth and distributed delay in birth process, J. Biol. Dyn. 8 (2014), 42-56.

[2] A. Tripathy, Oscillation criteria for a class of nonlinear fourth order neutral differential equations, Math. Slovaca 63 (2013), 243-262.

[3] Y. Kuang, Delay Differential Equations with Applications in Population Dynamics, Academic Press, New York, 1993.

[4] Z. Cheng, J. Ren, Existence of periodic solution for fourth-order Liénard type p-Laplacian generalized neutral differential equation with variable parameter, J. Appl. Anal. Comput. 5 (2015), 704-720.

[5] B. Du, Periodic solution to $p$-Laplacian neutral Liénard type equation with variable parameter, Math. Slovaca 63 (2013), 381-395.

[6] K. Wang, Y. Zhu, Periodic solutions for a fourth-order p-Laplacian neutral functional differential equation, J. Franklin Institute 347 (2010), 1158-1170.

[7] T. Xiong, R. Yuan, Existence of periodic solutions for $p$-Laplacian neutral functional equation with multiple deviating arguments, Topol. Methods Nonlinear Anal. 37 (2011), 235-258.

[8] A. Ardjouni, A. Rezaiguia, A. Djoudi, Existence of positive periodic solutions for fourth-order nonlinear neutral differential equations with variable delay, Adv. Nonlinear Anal. 3 (2014), 157-163.

[9] T. Candan, Existence of positive periodic solutions of first order neutral differential equations with variable coefficients, Appl. Math. Lett. 52 (2016), 142-148.

[10] W. Han, J. Ren, Some results on second-order neutral functional differential equations with infinite distributed delay, Nonlinear Anal. 70 (2009), 1393-1406.

[11] J. Ren, L. Yu, S. Siegmund, Bifurcations and chaos in a discrete predator-prey model with Crowley-Martin functional response, Nonlinear Dyn. 90 (2017), 19-41.

[12] J. L. Ren, Z. B. Cheng, S. Siegmund, Neutral operator and neutral differential equation, Abst. Appl. Anal. 2011 (2011), Article ID 969276.

[13] H. Wang, Positive periodic solutions of functional differential equations, J. Differential Equations 202 (2004), $354-366$.

[14] J. Wu, Z. Wang, Two periodic solutions of second-order neutral functional differential equations, J. Math. Anal. Appl. 329 (2007), 677-689.

[15] F. Kong, S. Lu, Z. Liang, Existence of positive periodic solutions for neutral Liénard differential equations with a singularity, Electron. J. Differential Equations 242 (2015), 1-12.

[16] Y. Xin, Z. Cheng, Study on a kind of neutral Rayleigh equation with singularity, Bound. Value Probl. 2017 (2017), Article ID 92 .

[17] F. Kong, S. Lu, Existence of positive periodic solutions of fourth-order singular $p$-Laplacian neutral functional differential equations, Filomat 31 (2017), 5855-5868.

[18] R. Gaines, J. Mawhin, Coincidence Degree and Nonlinear Differential Equation, Springer, Berlin, 1977.

[19] S. Peng, Periodic solutions for p-Laplacian neutral Rayleigh equation with a deviating argument, Nonlinear Anal. 69 (2008) 1675-1685.

[20] P. Torres, Z. B. Cheng, J. L. Ren, Non-degeneracy and uniqueness of periodic solutions for $2 n$-order differential equation, Discrete Contin. Dyn. Syst. A 33 (2013) 2155-2168. 